\title{
Discretization effects and gauge independence for the electric and magnetic screening masses*
}

\author{
Attilio Cucchieri and Frithjof Karsch ${ }^{\mathrm{a}}$ \\ ${ }^{\text {a} F a k u l t a ̈ t ~ f u ̈ r ~ P h y s i k, ~ U n i v e r s i t a ̈ t ~ B i e l e f e l d, ~ D-33615 ~ B i e l e f e l d, ~ G E R M A N Y ~}$
}

\begin{abstract}
We evaluate the electric and magnetic screening masses from the long-distance behavior of the (temporal and spatial) gluon correlation functions, for pure $S U(2)$ gauge theory at finite temperature. In order to investigate the gauge dependence of the screening masses we consider seven different gauges. We also evaluate these masses using different definitions of the lattice gluon field, corresponding to discretization errors of different orders.
\end{abstract}

\section{INTRODUCTION}

The electric and magnetic screening masses can be obtained from the exponential decay of finitetemperature gluon correlation functions at large separations [1,2]. It is important to stress that, even though the gluon propagator is a gauge dependent quantity, the (pole) masses obtained from it are - within a wide class of gauges gauge invariant to arbitrary order in perturbation theory $[3]$.

Here we present a preliminary study of the gluon propagator at finite temperature, using different definitions of the lattice gluon field, and considering several different gauges, in order to check for discretization effects and gauge independence [2] of the screening masses.

\section{LATTICE SETUP}

We consider a standard Wilson action for $S U(2)$ lattice gauge theory in 4 dimensions, with periodic boundary conditions. (Details of notation and numerical simulations will be given in [4.) We evaluate the lattice gluon propagators

$D_{\mu \mu}(z) \equiv \frac{1}{3 V N_{T}} \sum_{x_{3}, b}\left\langle Q_{\mu}^{b}\left(x_{3}+z\right) Q_{\mu}^{b}\left(x_{3}\right)\right\rangle$.

Here $V \equiv N_{1} N_{2} N_{3}$,

$Q_{\mu}^{b}\left(x_{3}\right) \equiv \sum_{x_{0}, x_{\perp}} A_{\mu}^{b}\left(x_{0}, x_{\perp}, x_{3}\right)$,

\footnotetext{
*Talk presented by A. Cucchieri. This work has been supported by the TMR network ERBFMRX-CT-970122 and the DFG under grant Ka 1198/4-1.
}

$A_{\mu}^{b}(x) \equiv \operatorname{Tr}\left[A_{\mu}(x) \sigma^{b}\right] / 2$, and $\sigma^{b}$ is a Pauli matrix. The lattice gauge field $A_{\mu}(x)$ will be defined in Section 2.2 below.

From the exponential decay at large separation of the (temporal and spatial) gluon correlation functions we can obtain the electric and magnetic screening masses [1,2]:

$\begin{aligned} D_{00}(z) & \sim \exp \left(-M_{e} z\right) \\ D_{i i}(z) & \equiv D_{11}(z)+D_{22}(z) \sim \exp \left(-M_{m} z\right) .\end{aligned}$

As mentioned before, these screening masses are expected to be gauge invariant [3].

\subsection{Gauge Fixing}

We consider the "so-called" $\lambda$-gauge condition [5], i.e. we look for a local minimum of the functional

$\mathcal{E}_{U}^{\lambda}[g] \equiv-\sum_{x, \mu} \operatorname{Tr}\left[\lambda_{\mu} g(x) U_{\mu}(x) g^{\dagger}\left(x+e_{\mu}\right)\right]$,

where $\lambda_{0}=\lambda$ and $\lambda_{i}=1$ for $i=1,2,3$. We have performed simulations with $\lambda=2,1,0.5,0.1,0.01$ and 0 . The case $\lambda=1$ is the Landau gauge, whereas $\lambda=0$ is the Coulomb gauge. In the last case we also impose the condition

$Q_{0}^{b}\left(x_{0}\right) \equiv \sum_{x_{\perp}, x_{3}} A_{0}^{b}\left(x_{0}, x_{\perp}, x_{3}\right)=\mathcal{Q}_{0}^{b}$,

which is automatically satisfyed for any $\lambda \neq 0$.

Finally, we consider the standard Maximally Abelian gauge. In this case we fix the residual $U(1)$-gauge symmetry by imposing a $U(1)$ Landau gauge condition [6]. 


\subsection{Discretization Effects}

The gauge field is usually defined as

$A_{\mu}^{(1)}(x) \equiv\left[U_{\mu}(x)-U_{\mu}^{\dagger}(x)\right] /(2 i)$,

where $U_{\mu}(x) \in S U(2)$ are link variables. We can also consider other definitions of $A_{\mu}(x)$, leading to discretization errors of different orders. For example, we can write [7,8]

$A_{\mu}^{(2)}(x) \equiv\left\{\left[U_{\mu}(x)\right]^{2}-\left[U_{\mu}^{\dagger}(x)\right]^{2}\right\} /(4 i)$

$A_{\mu}^{(3)}(x) \equiv\left\{\left[U_{\mu}(x)\right]^{4}-\left[U_{\mu}^{\dagger}(x)\right]^{4}\right\} /(8 i)$.

If we set $U_{\mu}(x) \equiv \exp \left[i a g_{0} \vec{\sigma} \cdot \overrightarrow{\mathcal{A}}(x)\right]$, we obtain that $A_{\mu}^{(1)}(x), A_{\mu}^{(2)}(x)$ and $A_{\mu}^{(3)}(x)$ are equal to $a g_{0} \vec{\sigma} \cdot \overrightarrow{\mathcal{A}}(x)$ plus terms of order $a^{3} g_{0}^{3}$. We can also consider [8]

$A_{\mu}^{(4)}(x) \equiv\left[4 A_{\mu}^{(1)}(x)-A_{\mu}^{(2)}(x)\right] / 3$

$A_{\mu}^{(5)}(x) \equiv\left[16 A_{\mu}^{(1)}(x)-A_{\mu}^{(3)}(x)\right] / 15$

$A_{\mu}^{(6)}(x) \equiv\left[4 A_{\mu}^{(2)}(x)-A_{\mu}^{(3)}(x)\right] / 3$

$A_{\mu}^{(7)}(x) \equiv\left[64 A_{\mu}^{(1)}(x)-20 A_{\mu}^{(2)}(x)+A_{\mu}^{(3)}(x)\right] / 45$.

It is easy to check that $A_{\mu}^{(4)}(x), A_{\mu}^{(5)}(x)$ and $A_{\mu}^{(6)}(x)$ are equal to $a g_{0} \vec{\sigma} \cdot \overrightarrow{\mathcal{A}}(x)$ plus terms of order $a^{5} g_{0}^{5}$, and that $A_{\mu}^{(7)}(x)=a g_{0} \vec{\sigma} \cdot \overrightarrow{\mathcal{A}}(x)$ plus terms of order $a^{7} g_{0}^{7}$. Finally, by writing $U_{\mu}(x) \equiv U_{\mu}^{0}(x) \mathbb{1}+i \vec{\sigma} \cdot \vec{U}_{\mu}(x)$, one can define the "perfect" gluon field [6,9]

$A_{\mu}^{(p) b}(x) \equiv \frac{U_{\mu}^{b}(x)}{\left\|\vec{U}_{\mu}(x)\right\|} \arctan \left(\frac{\left\|\vec{U}_{\mu}(x)\right\|}{U_{\mu}^{0}(x)}\right)$,

which is equal to $a g_{0} \vec{\sigma} \cdot \overrightarrow{\mathcal{A}}(x)$ with no discretization errors.

Let us notice that the minimizing condition defined in Eq. (11) implies

$$
\sum_{\mu=1}^{4} \lambda_{\mu}\left[\mathcal{A}_{\mu}^{b}(x)-\mathcal{A}_{\mu}^{b}\left(x-e_{\mu}\right)\right]=\mathcal{O}\left(a^{2} g_{0}^{2}\right) \text {. }
$$

\section{SIMULATIONS AND RESULTS}

We have evaluated the gluon propagators $D_{i i}^{(n)}(z)$ and $D_{00}^{(n)}(z)$, using the eight different definitions of the gluon field $A_{\mu}^{(n)}(x)$ given in the previous section, for the seven gauges considered here, with two different sets of parameters: (s1) $N_{T}=4, V=12^{2} \times 24, \beta=2.512$, and (s2) $N_{T}=8, V=16^{2} \times 32, \beta=2.74$. (In both cases $T \approx 2 T_{c}$.) We note that we have generated different sets of configurations for each different gauge, while, in a given gauge, the different lattice discretizations of the gluon propagators have been evaluated using the same set of configurations.

We have found that, in all gauges, the eight gluon correlation functions $D^{(n)}(z)$ are equal modulo a constant factor, both in the electric and in the magnetic sector. 1 (In Fig. 11 we plot, as an example, the data for the magnetic case in Landau gauge.) This result clearly implies that the screening masses are independent of the discretization of the gluon field.

Since the different lattice discretizations of the gluon propagator differ only by a constant factor, we can consider any of them [for example $\left.D^{(1)}(z)\right]$ in order to compare the results obtained in different gauges. We then find that, in the magnetic sector, the gluon correlation functions $D(z)$ corresponding to different gauges are equal modulo a constant factor (see Fig. 2, top figure). This clearly implies that the magnetic mass obtained in this way is gauge independent, at least within the seven gauges considered here. On the contrary, in the electric sector, the propagators corresponding to the gauges $\lambda=0.01$ and 0 , are not proportional to the propagators obtained in the other gauges (see Fig. 2, middle figure, symbols $*$ and $\times$ ). Moreover, the discrepancy between the Coulomb gauge propagator and the propagators in the $\lambda$-gauges does not decrease as one gets closer to the continuum limit (see Fig. 2, bottom figure). However, the strong variation seen in the propagator for the case $\lambda=0.01$ (see Fig. 2, middle and bottom figures) makes us expect that the "gauge dependence" seen for this small $\lambda$-value, as well as for $\lambda=0$, may be related

\footnotetext{
${ }^{1} \mathrm{~A}$ similar result has also been obtained at zero temperature 7. Actually, for $\lambda=0.01$ and 0 , we have found that the propagator $D_{00}^{(3)}(z)$ is not proportional to the other seven propagators $D_{00}^{(n)}(z)$. However, this discrepancy seems to decrease - as expected - if one gets closer to the continuum limit, i.e. going from the $\mathbf{s} \mathbf{1}$ to the $\mathbf{s} 2$ simulation 舞.
} 
to our gauge-fixing procedure, rather than indicating a gauge dependence of the electric mass. In fact, we observe that the gauge-fixing procedure does not always bring the $U_{0}(x)$ fields close to unity, which is needed to ensure a meaningful definition of the $A_{0}(x)$ field in terms of the compact gauge-field variables. We are currently investigating this issue in more detail [4].

\section{REFERENCES}

1. J. E. Mandula and M. Ogilvie, Phys. Lett. B201, 117 (1988); U. M. Heller et al., Phys. Rev. D57, 1438 (1998).

2. F. Karsch et al., hep-ph/9809414; P. Petreczky, these Proceedings.

3. R. Kobes et al., Phys. Rev. Lett. 64, 2992 (1990); Nucl. Phys. B355, 1 (1991).

4. A. Cucchieri and F. Karsch, in preparation.

5. C. Bernard et al., Nucl. Phys. B (Proc. Suppl.) 17, 593 (1990).

6. K. Amemiya and H. Suganuma, hep-lat 9811035 .

7. L. Giusti et al., Phys. Lett. B432, 196 (1998).

8. A. Cucchieri and T. Mendes, hep-lat 9902024; and in preparation; A. Cucchieri, hep-lat/9908050.

9. H. Nakajima and S. Furui, Nucl. Phys. B (Proc. Suppl.) 73, 865 (1999).

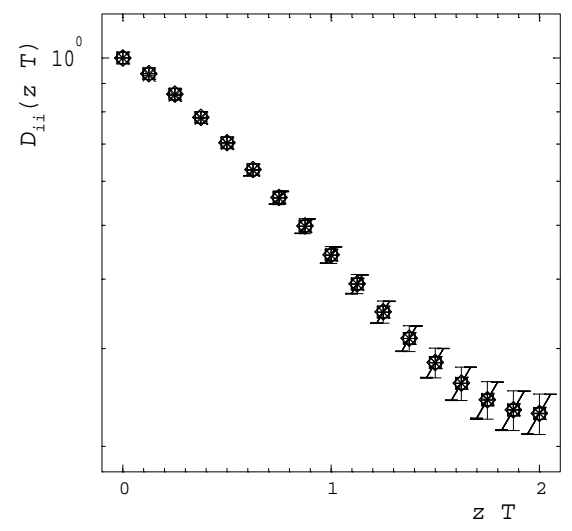

Figure 1. Data for the eight different discretizations of the gluon propagator $D_{i i}(z T)$ in Landau gauge with simulation parameters $\mathbf{s}$. The propagators are normalized to 1 at $z T=0$. Error bars are one standard deviation.
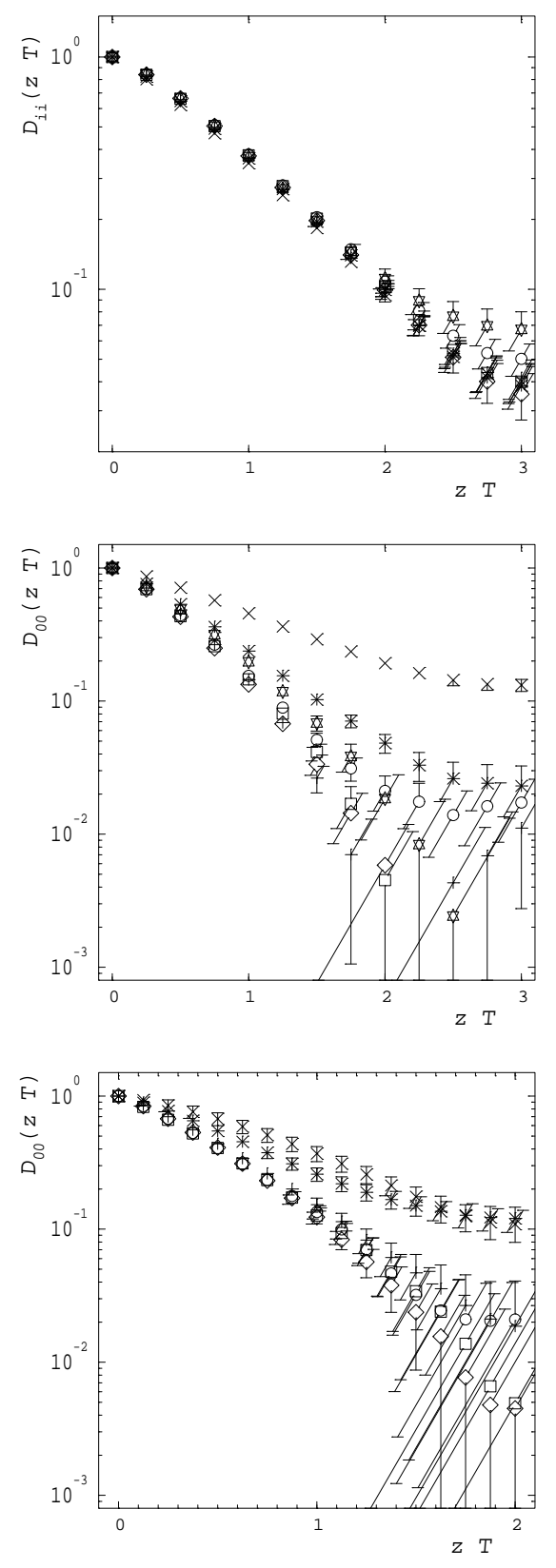

Figure 2. Data for the $D^{(1)}$ gluon propagator in the seven different gauges considered here: $D_{i i}(z T)$ in Landau gauge with simulation parameters s1 (top figure); $D_{00}(z T)$ in Coulomb gauge with simulation parameters $\mathbf{s} \mathbf{1}$ (center figure), and with simulation parameters s2 (bottom figure). The propagators are normalized to 1 at $z T=0$. Error bars are one standard deviation. 